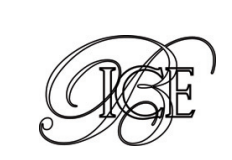

Esmeralda Linares Navarro*

\title{
LA IMPLEMENTACIÓN DE ESTRATEGIAS DE OFFSHORING EN EMPRESAS DEL ESTE DE EUROPA Una visión distinta de la deslocalización en Europa oriental
}

Los países de Europa del Este han sido el destino europeo preferido por muchas empresas para deslocalizar sus actividades. Sin embargo, en los últimos años las empresas de estos países están aprovechando las oportunidades derivadas del uso de estrategias de offshoring. En este sentido, el objetivo de este trabajo es ofrecer una visión, poco estudiada hasta el momento, de la deslocalización en Europa del Este; analizando las principales características de las estrategias de offshoring implementadas por empresas de estos países, a partir de los datos de Eurostat.

Palabras clave: offshoring, estrategia, internacionalización, deslocalización, Europa del Este. Clasificación JEL: L23, M16.

\section{Introducción}

El offshoring ha sido una de las estrategias más utilizadas por las empresas europeas en los últimos años con el objetivo de mantener una posición competitiva en un mercado cada vez más globalizado. Una de las principales razones por las que las empresas implementan estas estrategias es, sin duda alguna, el ahorro en costes que supone localizar toda o parte de la producción en países con menores costes productivos, particularmente costes laborales, consiguiendo de este modo una mayor eficiencia (Roza, et al., 2011). Los países asiáticos, principalmente China e India, han sido los grandes protagonistas de la deslocalización de las actividades de empresas de medio mundo. Sin embargo, dentro de Europa, países como Polonia, Bulgaria, Lituania o Eslovaquia, también han estado vinculados a este fenómeno. Los

* Departamento de Dirección de Empresas. Facultad de Economía. Universitat de València.

Versión de enero de 2015. países de Europa del Este han sido el destino elegido por muchas empresas europeas, y de otras partes del mundo, para deslocalizar algunas de sus actividades (AT Kearney, 2014; Marin, 2006; Sass y Fifekova, 2011). Sin embargo, en los últimos años algunos de estos países están participando en la deslocalización en primera persona y de una forma más activa, es decir, sus empresas están empezando a aprovechar las oportunidades que supone deslocalizar actividades hacia otros países. En este sentido, el objetivo de este trabajo es ofrecer una visión de este fenómeno poco estudiada hasta el momento: la implementación de estrategias de offshoring por parte de empresas del este de Europa. Utilizando los datos de la encuesta sobre International Sourcing incluida en la base de datos Structural Business Statistics (SBS) de Eurostat, se analizan aquellos países de Europa del este que están adquiriendo protagonismo en el uso de estas estrategias, así como sus principales características en estos países: qué tipo de actividades funciones están siendo $D$ 
deslocalizadas por sus empresas, que modalidad de offshoring prefieren, qué razones les llevan a implementar estas estrategias, cuáles son los principales destinos elegidos por estas empresas para llevarlas a cabo y qué efectos está generando el uso de las mismas en los niveles de empleo de su país.

Para ello, el trabajo se estructura de la siguiente manera. A continuación, se definen -desde el punto de vista teórico- las estrategias de offshoring, así como sus principales ventajas e inconvenientes. En el tercer apartado se realiza un análisis descriptivo en profundidad del uso de estas estrategias en los países de Europa del Este. Finalmente, se presentan las principales conclusiones y limitaciones de este estudio.

\section{Estrategias de offshoring}

En los últimos años las estrategias de offshoring han adquirido una gran importancia en el ámbito académico, derivada de su creciente implementación en el ámbito empresarial. Esta opción estratégica, que ha sido considerada por algunos autores como decisiva para mantener y mejorar la competitividad de las empresas, tanto a nivel nacional como internacional (Amesse et al., 2001), posee una amplia variedad de definiciones y ha sido analizada desde distintos puntos de vista. El término offshoring no es nuevo, aunque con frecuencia se emplea para describir estrategias diferentes. Algunas investigaciones previas lo definen como el desplazamiento de procesos y/o actividades a otros países (Levy, 2005). Sin embargo, ese traslado de actividades y/o procesos al extranjero puede llevarse a cabo de formas distintas. La empresa puede subcontratar dichas actividades a empresas independientes ubicadas en un país extranjero (external offshoring) o bien puede realizar dichas actividades a través de una filial propia que se encuentre localizada en otro país (internal offshoring). Empleamos el término external offshoring como sinónimo de otros conceptos utilizados en la literatura para referirse a la subcontratación internacional de actividades a través de terceros como son non-captive offshoring (WTO, 2005) y offshore outsourcing (Pyndt y Pedersen, 2006); y el término internal offshoring para referirnos a la deslocalización de actividades a través de filiales propias, definido por otros autores como captive offshoring (Pyndt y Pedersen, 2006), offshore inhouse sourcing (OECD, 2007) u offshore insourcing (Kotabe et al., 2008).

Cualquiera que sea la modalidad empleada, para la mayoría de empresas las estrategias de offshoring son una forma rápida y eficaz de reducir costes. La principal ventaja que se asocia al uso de estas estrategias es el ahorro en costes que les supone a las empresas que las implementan. Sin embargo, pueden existir ventajas de carácter estratégico que, además de reducir costes, permiten centrarse en las actividades que son competencias esenciales, así como adquirir una mayor flexibilidad y un mejor aprovechamiento de las capacidades de proveedores especializados, o conseguir una calidad mayor a la obtenida por la propia empresa (Dess et al., 1995; Kotabe y Murray, 1990; Quinn, 1992). Las empresas que hacen un uso estratégico del offshoring pueden convertirse en organizaciones focalizadas, simplificadas y ágiles (Pagnoncelli, 1993).

El uso de estas estrategias también puede presentar riesgos para la organización. Algunos estudios plasman el creciente interés por estudiar los factores de riesgo asociados al offshoring (Kotabe y Murray, 2004; Zsidish, 2003). El incremento por parte de los Gobiernos de las medidas legales internacionales puede suponer un riesgo potencial para cualquier organización (Lisle, 2003). Igualmente, la variabilidad del tipo de cambio, la inestabilidad política, social y/o legal, y las diferencias culturales, sociales y/o legales, son algunos de los principales riesgos asociados a la estrategia (Schniederjans y Zuckweiler, 2004). Además de riesgos externos, dentro de la propia organización pueden surgir ciertos factores de riesgo. Entre ellos destacan la reacción negativa de los empleados $\triangleright$ 
LA IMPLEMENTACIÓN DE ESTRATEGIAS DE OFFSHORING EN EMPRESAS DEL ESTE DE EUROPA...

\begin{tabular}{|c|c|c|c|c|c|}
\hline \multicolumn{6}{|c|}{$\begin{array}{c}\text { TABLA } 1 \\
\text { GLOBAL SERVICES LOCATION INDEX (GSLI) - } 2014\end{array}$} \\
\hline № Ranking & País & GSLI & № Ranking & País & GSLI \\
\hline 1 & India & 7,04 & 26 & Bangladesh & 5,34 \\
\hline 2 & China & 6,15 & 27 & UK & 5,34 \\
\hline 3 & Malasia & 5,98 & 28 & Túnez & 5,30 \\
\hline 4 & México & 5,90 & 29 & Ghana & 5,30 \\
\hline 5 & Indonesia & 5,87 & 30 & Panamá & 5,28 \\
\hline 6 & Tailandia & 5,87 & 31 & Hungría & 5,28 \\
\hline 7 & Filipinas & 5,75 & 32 & España & 5,28 \\
\hline 8 & Brasil & 5,69 & 33 & República Checa & 5,25 \\
\hline 9 & Bulgaria & 5,62 & 34 & Marruecos & 5,25 \\
\hline 10 & Egipto & 5,62 & 35 & Eslovaquia & 5,19 \\
\hline 11 & Polonia & 5,54 & 36 & Islas Mauricio & 5,14 \\
\hline 12 & Vietnam & 5,54 & 37 & Canadá & 5,13 \\
\hline 13 & Chile & 5,53 & 38 & Argentina & 5,06 \\
\hline 14 & USA & 5,53 & 39 & Turquía & 5,05 \\
\hline 15 & Lituania & 5,52 & 40 & Senegal & 5,04 \\
\hline 16 & Sri Lanka & 5,51 & 41 & Ucrania & 5,03 \\
\hline 17 & Alemania & 5,46 & 42 & Uruguay & 4,91 \\
\hline 18 & Rumanía & 5,45 & 43 & Colombia & 4,90 \\
\hline 19 & Emiratos Árabes & 5,39 & 44 & Portugal & 4,89 \\
\hline 20 & Jordán & 5,39 & 45 & Jamaica & 4,84 \\
\hline 21 & Rusia & 5,38 & 46 & Francia & 4,84 \\
\hline 22 & Estonia & 5,38 & 47 & Sudáfrica & 4,79 \\
\hline 23 & Letonia & 5,35 & 48 & Singapur & 4,68 \\
\hline 24 & Costa Rica & 5,35 & 49 & Australia & 4,65 \\
\hline \multirow[t]{2}{*}{25} & Pakistán & 5,34 & 50 & Israel & 4,52 \\
\hline & & & 51 & Irlanda & 4,36 \\
\hline
\end{tabular}

frente al offshoring, la falta de apoyo de la alta dirección para llevar a cabo dicha estrategia o la elección inadecuada de los miembros del equipo que la llevará a cabo.

Aunque los riesgos e inconvenientes asociados al uso de estas estrategias existen y son relevantes, para la mayoría de las empresas continúan siendo una opción estratégica clave que les permite mantener, o incluso mejorar, su posición competitiva en un mercado cada vez más globalizado.

\section{El offshoring en Europa del Este}

El uso de estrategias de offshoring se ha vinculado tradicionalmente a actividades productivas intensivas en mano de obra y que añaden poco valor al producto, por lo que resulta más económico, eficaz y competitivo deslocalizar dichas actividades hacia países intensivos en mano de obra -fundamentalmente de baja cualificación-y con salarios bajos. Del Global Service Location Index (GSLI) elaborado por AT Kearney en $2014^{1}$ se desprende que Asia continúa siendo la región preferida por la mayoría de las empresas para deslocalizar sus actividades (Tabla 1). De entre los países asiáticos, India y China son el principal destino de la deslocalización de empresas de medio mundo.

Los niveles salariales de los países de destino suele ser un elemento relevante para su elección. Sin embargo, en el uso de estrategias de offshoring, existen otros factores que influyen en la misma (Jensen y Pedersen, 2010). Entre estos $\square$

\footnotetext{
1 EI GSLI-2014, elaborado desde 2004 por AT Kearney, analiza y clasifica los 51 mejores países del mundo para realizar offshoring. Para calcular este índice se evalúan 25 medidas agrupadas en tres categorías distintas: 1) atractivo financiero (40 por 100), 2) habilidades y disponibilidad de la mano de obra (30 por 100) y 3) entorno empresarial del país (30 por 100) (AT Kearney, 2014).
} 
factores destaca el nivel de infraestructuras que tenga el país, la disponibilidad de la mano de obra necesaria, la presencia de una red de empresas con las que exista un vínculo, o el idioma y las barreras culturales (Eurostat, 2009). En conjunto, todos estos factores han llevado a las empresas, particularmente europeas, a considerar otras regiones más próximas -cultural y geográficamente- $y$ con los recursos necesarios -mano de obra disponible, salarios bajos, infraestructuras eficientes, etcétera- para llevar a cabo sus estrategias de offshoring. En este sentido, Europa del Este es también una de las regiones clave en la deslocalización de actividades. Como puede observarse en la Tabla 1, entre los 51 mejores países del mundo para realizar offshoring podemos encontrar 9 países del este de Europa: Bulgaria (9), Polonia (11), Lituania (15), Rumanía (18), Estonia (22), Letonia (23), Hungría (31), República Checa (33) y Eslovaquia (35); de los cuales 6 se encuentran entre los 25 mejores países del mundo para realizar dichas estrategias.

Esta región europea posee una combinación de elementos que la hacen atractiva para la deslocalización: países con una industria madura y altos niveles de capital humano, como Polonia, destino de estrategias de offshoring de multinacionales como Sabre, Motorola o Capgemini; así como países emergentes donde los costes son más bajos y la industria y el escenario regulatorio están en desarrollo, como Bulgaria, destino de centros de tecnología de la información avanzada centrados principalmente en el desarrollo de software tradicional como es el caso de CSC y SAP (AT Kearney, 2014).

Por todo ello, estos países han sido el destino de las estrategias de offshoring de numerosas empresas -europeas y de otras partes del mundo- y el objeto de análisis de algunas investigaciones (Marin, 2006; Sass y Fifekova, 2011; Stare y Rubalcaba, 2009).

No obstante, esta región europea no sólo es receptora de actividades deslocalizadas por empresas de otros países, sino que las empresas de esta región también han sabido aprovechar las ventajas de la deslocalización. Las investigaciones realizadas hasta el momento se han centrado en analizar la región de Europa del Este desde la perspectiva de «destino del offshoring», dejando de lado la perspectiva de «origen del offshoring», es decir, el análisis del uso de estas estrategias por parte de las empresas localizadas en esta región. Sin embargo, existen datos que demuestran la implementación de estas estrategias por parte de empresas localizadas en estos países; y por tanto, analizarlos ayudará a conocer las principales características asociadas a su uso y ofrecerá una visión distinta, a la tradicionalmente mostrada, de la deslocalización en Europa del Este.

Una de las pocas bases de datos que ofrece información a nivel europeo sobre las estrategias de offshoring implementadas por las empresas es la Structural Business Statistics de Eurostat. A través de la encuesta International Sourcing Survey, esta base de datos incluye información relativa al uso de estas estrategias en empresas europeas. La última encuesta de la que se disponen datos se realizó en 2012 para un total de 15 países europeos entre los que se encuentran 6 países del este de Europa: Bulgaria, Eslovaquia, Estonia, Letonia, Lituania y Rumanía. En total la encuesta recoge información sobre el uso de estrategias de offshoring durante el periodo 2009-2011 de más de 40.000 empresas europeas con 100 o más empleados, de sectores industriales y de servicios ${ }^{2}$. Las empresas de Europa del Este incluidas en la base de datos representan casi la cuarta parte del total de empresas encuestadas (Tabla 2).

En esta misma tabla se observa cómo el porcentaje de empresas que realizan estrategias de offshoring en países de Europa del Este es de 4,03 por 100 , mientras que en otros países europeos este dato se sitúa en el 10,50 por 100. Aunque los valores calculados para ambos grupos de países $\triangle$

\footnotetext{
2 En 2012, International Sourcing Survey utiliza la clasificación de actividades NACE Rev. 2 incluyendo los sectores de actividad comprendidos entre las secciones $\mathrm{B}$ y $\mathrm{N}$, exceptuando la sección $\mathrm{K}$ (actividades financieras y de seguros).
} 


\begin{tabular}{|c|c|c|c|}
\hline País de origen & $\mathrm{N}^{\circ}$ empresas encuestadas & $\mathrm{N}^{\circ}$ empresas con offshoring & Porcentaje empresas con offshoring \\
\hline $\begin{array}{c}\text { Países de Europa del Este } . . . . . . . . . . . \\
\text { Bulgaria }\end{array}$ & $\begin{array}{r}10.037 \\
2.256\end{array}$ & $\begin{array}{r}404 \\
24\end{array}$ & $\begin{array}{l}4,03 \\
1,06\end{array}$ \\
\hline 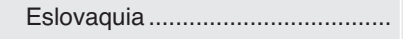 & 1.277 & 141 & 11,04 \\
\hline Estonia & 514 & 45 & 8,75 \\
\hline Letonia ............................................... & 600 & 41 & 6,83 \\
\hline Lituania ........................................... & 945 & 7 & 0,74 \\
\hline Rumanía ....................................... & 4.445 & 146 & 3,28 \\
\hline Otros países europeos ……........... & 30.279 & 3.180 & 10,50 \\
\hline Bélgica.......................................... & 2.216 & 363 & 16,38 \\
\hline Dinamarca ....................................... & 1.244 & 314 & 25,24 \\
\hline Finlandia ........................................... & 1.198 & 247 & 20,62 \\
\hline Francia.............................................. & 13.543 & 800 & 5,91 \\
\hline 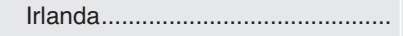 & 1.259 & 148 & 11,76 \\
\hline Noruega & 1.431 & 172 & 12,02 \\
\hline Países Bajos..................................... & 4.560 & 447 & 9,80 \\
\hline Portugal .............................................. & 2.496 & 381 & 15,26 \\
\hline Suecia.......................................... & 2.332 & 308 & 13,21 \\
\hline Total & 40.316 & 3.584 & 8,89 \\
\hline
\end{tabular}

(Europa del Este vs. otros países europeos) indican grandes diferencias en el uso de estrategias de offshoring, se puede observar cómo a nivel de país, Eslovaquia, Estonia o Letonia presentan niveles considerables, y además muy próximos a los mostrados por otros países europeos como Francia, Irlanda, Noruega, Países Bajos o Suecia.

En este sentido, resulta conveniente analizar en profundidad los datos procedentes de la International Sourcing Survey para conocer y estudiar las principales características del uso de estrategias de offshoring por parte de las empresas de los países del Este, consiguiendo de este modo avanzar en la investigación del fenómeno del offshoring en Europa oriental.

\subsection{Análisis del uso de estrategias de offshoring en empresas de países del Este}

Tal y como se menciona en el apartado anterior, las ventajas derivadas del offshoring han llevado a numerosas empresas a deslocalizar sus actividades en distintas partes del mundo. Los países de Europa del Este, elegidos por muchas de estas empresas para la deslocalización de sus actividades, albergan además un número considerable de empresas que utilizan estas estrategias como una posible vía para mantener su ventaja competitiva. En este apartado analizaremos las características que presentan las empresas del este de Europa en cuanto al uso del offshoring (sectores de actividad a los que pertenecen, modos utilizados, tipos de actividades/funciones deslocalizadas, motivos/razones para su uso, principales destinos, etcétera); identificando posibles diferencias entre los países estudiados.

\subsubsection{Offshoring vs. sector de actividad}

En general, las empresas manufactureras suelen ser las más propensas en el uso de estrategias de offshoring. En la mayoría de los países europeos, aproximadamente dos tercios de las empresas que realizan offshoring pertenecen a este, mientras que sólo un tercio corresponde a otros sectores, entre los que se encuentra el sector servicios (Eurostat, 2013).

En la Tabla 3, en la página siguiente, en la que se recoge el porcentaje de empresas en Europa del Este que utilizan estrategias de offshoring según el sector de actividad, se observa cómo -al igual que ocurre en el conjunto de Europa- la mayoría $\triangle$ 


\begin{tabular}{|c|c|c|c|}
\hline País de origen & $\mathrm{N}^{\circ}$ empresas con offshoring & Sector industrial (porcentaje) & Otros sectores (porcentaje) \\
\hline Países de Europa del Este ............ & 404 & 54,46 & 45,30 \\
\hline Bulgaria & 24 & 66,67 & 33,33 \\
\hline Eslovaquia............................................. & 141 & 51,77 & 48,23 \\
\hline 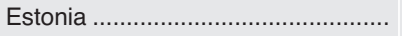 & 45 & 80,00 & 17,78 \\
\hline 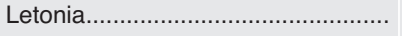 & 41 & 41,46 & 58,54 \\
\hline Lituania & 7 & 0,00 & 100,00 \\
\hline Rumanía.................................... & 146 & 53,42 & 46,58 \\
\hline
\end{tabular}

son empresas manufactureras pertenecientes al sector industrial $(54,46$ por 100$)$. Sin embargo, en esta región el porcentaje de empresas de otros sectores $^{3}$ que implementan offshoring es ligeramente superior a lo habitual $(45,30$ por 100$)$.

No obstante, los datos a nivel de país presentan algunas particularidades que conviene destacar. Mientras que en Bulgaria, Eslovaquia, Estonia y Rumanía las empresas manufactureras son más proclives a utilizar offshoring que las empresas de otros sectores, con importantes diferencias en algunos de estos países como es el caso de Bulgaria $(66,67$ por 100 frente al 33,33 por 100 ) o Estonia (80 por 100 frente al 17,78 por 100 ); en Letonia y Lituania las empresas del sector servicios son más activas en el uso de estas estrategias que las empresas manufactureras.

\subsubsection{Modalidad de offshoring}

Como se ha descrito en el apartado 2, la estrategia de offshoring puede implementarse utilizando distintas modalidades. Las empresas pueden llevar a cabo external offshoring (EOF) deslocalizando las actividades en otros países a través de empresas totalmente independientes, es decir, con las que no se tenga ningún vínculo de propiedad; o bien pueden utilizar internal offshoring (IOF) en cuyo caso las actividades deslocalizadas serán realizadas por una filial de la propia empresa que esté ubicada en otro país.

\footnotetext{
3 Incluye el sector servicios, exceptuando actividades financieras y de seguros.
}

En el Gráfico 1 se puede observar que tanto las empresas de países del este de Europa como las empresas de los otros países europeos analizados prefieren utilizar estrategias de offshoring de carácter interno, es decir IOF, en ambos casos, de casi el 60 por 100. En cuanto al uso de EOF, el porcentaje de empresas de países de Europa del Este que utilizan esta modalidad es superior al de las empresas de otros países europeos (44,31 vs. $36,98$ por 100$)$.

Sin embargo, dentro de los países del Este, existen algunas diferencias importantes en el uso de una modalidad de offshoring $u$ otra. Mientras que la mayoría de las empresas búlgaras, eslovacas y lituanas que realizan offshoring lo hacen a través de IOF $(66,67 ; 78,01$ y 85,71 por 100 , respectivamente); las empresas estonias y letonas prefieren utilizar EOF para llevar a cabo el offshoring (64,44 y 73,17 por 100, respectivamente). Destaca el caso de Lituania en el que casi el 86 por 100 de las empresas utiliza IOF y sólo un 28,57 por 100 EOF. Por último, se observa cómo en Rumanía no existen preferencias por una modalidad u otra, ya que el porcentaje de empresas rumanas que implementa IOF $(38,36$ por 100$)$ es similar al de las empresas que implementan EOF $(37,67$ por 100$)$.

\subsubsection{Offshoring vs. tipo de actividad deslocalizada}

Originalmente el offshoring se concibió como la estrategia a utilizar por las empresas manufactureras que querían trasladar parte de su producción al exterior; sin embargo, los avances en $\triangleright$ 


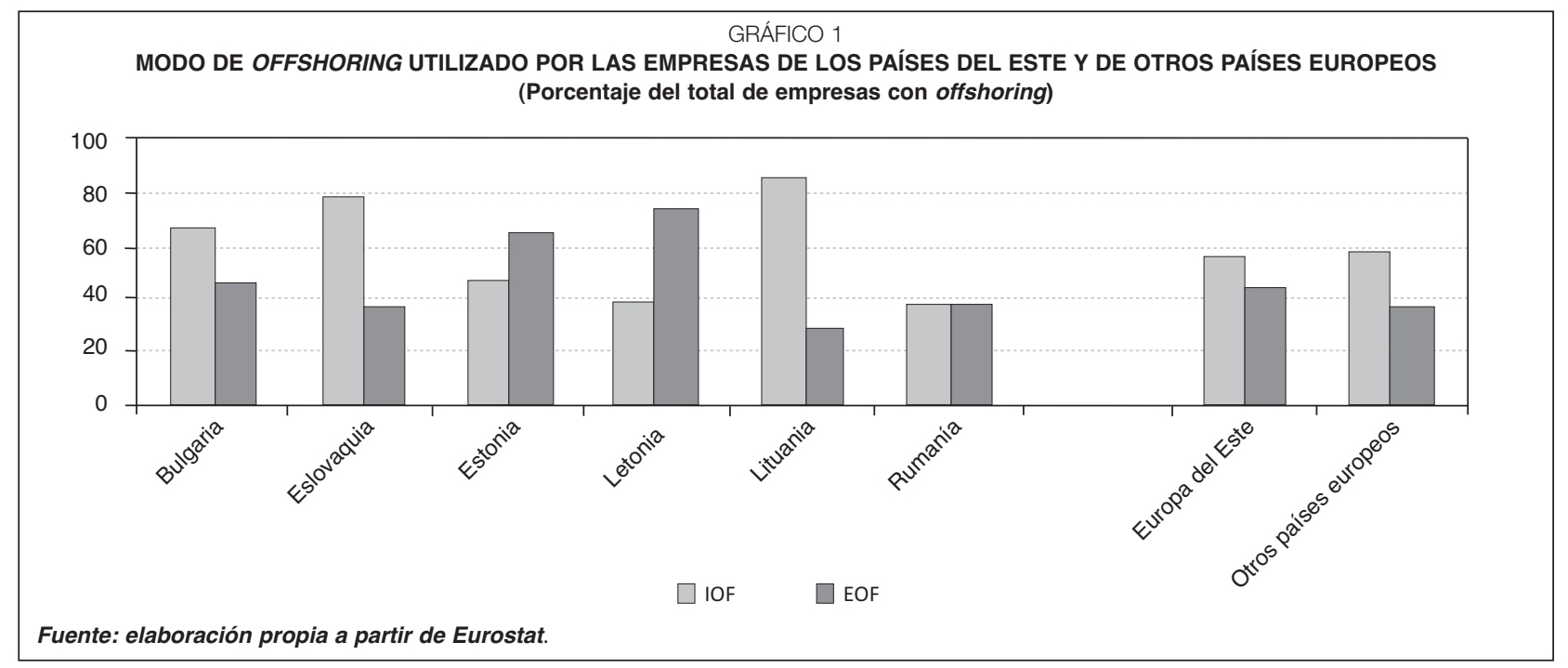

\begin{tabular}{|c|c|c|c|}
\hline \multicolumn{4}{|c|}{$\begin{array}{c}\text { TABLA } 4 \\
\text { PORCENTAJE DE EMPRESAS DE EUROPA DEL ESTE QUE UTILIZAN ESTRATEGIAS DE OFFSHORING SEGÚN LA ACTIVIDAD } \\
\text { DESLOCALIZADA (PRINCIPAL VS. SECUNDARIA) }\end{array}$} \\
\hline País de origen & $\mathbf{N}^{\circ}$ empresas con offshoring & $\begin{array}{l}\text { Actividad principal } \\
\text { (porcentaje) }\end{array}$ & $\begin{array}{l}\text { Actividades secundarias } \\
\text { (porcentaje) }\end{array}$ \\
\hline Países de Europa del Este .............. & 404 & 38,37 & $\mathbf{7 8 , 4 7}$ \\
\hline Bulgaria & 24 & 58,33 & 58,33 \\
\hline Eslovaquia........................................... & 141 & 36,17 & 82,27 \\
\hline Estonia & 45 & 44,44 & 77,78 \\
\hline 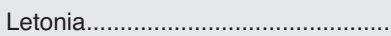 & 41 & 53,66 & 90,24 \\
\hline Lituania & 7 & 57,14 & 85,71 \\
\hline Rumanía & 146 & 30,14 & 74,66 \\
\hline
\end{tabular}

digitalización y codificación de servicios han Ilevado a que sea utilizada no sólo para trasladar actividades productivas sino también de servicios; así como a ser utilizada cada vez más por empresas del sector servicios (Eurostat, 2013).

En las empresas se pueden distinguir dos tipos de actividades/funciones: 1) las relacionadas directamente con la actividad principal de la empresa (core activitiy) y que se definen como la producción de bienes o servicios finales realizada por la empresa, destinada al mercado o a terceros y que genera ingresos. En la mayoría de los casos, la core activity coincide con la actividad principal de la empresa, sin embargo puede incluir otras actividades secundarias consideradas por la empresa como parte de sus funciones básicas; y 2) las actividades/funciones secundarias o de apoyo a la actividad principal (support actitivies) y que se realizan para permitir/facilitar la producción de bienes o servicios. Los outputs de estas actividades no son destinados directamente al mercado ni a terceros, son utilizados por la propia empresa ${ }^{4}$.

En general, las empresas utilizan el offshoring para deslocalizar support activities; mientras que las core activities no suelen ser objeto de deslocalización. No obstante, es cada vez más frecuente la deslocalización de actividades que resultan esenciales para la ventaja competitiva de la empresa (Linares-Navarro et al., 2014).

En los países de Europa del Este las empresas utilizan en mayor medida estrategias de offshoring para deslocalizar actividades secundarias (Tabla 4). Más del 78 por 100 de las empresas de estos países que realizan offshoring lo hace para deslocalizar support activities, mientras que cerca del 38 por 100 lo utiliza para deslocalizar core activities. $\triangleright$

4 Las support activities incluyen: distribución y logística, actividades comerciales (marketing, ventas y servicios post-venta), servicios de tecnologías de la información y comunicación (TIC), actividades administrativas y de management, ingeniería y servicios técnicos, o investigación y desarrollo (I+D). 
TABLA 5

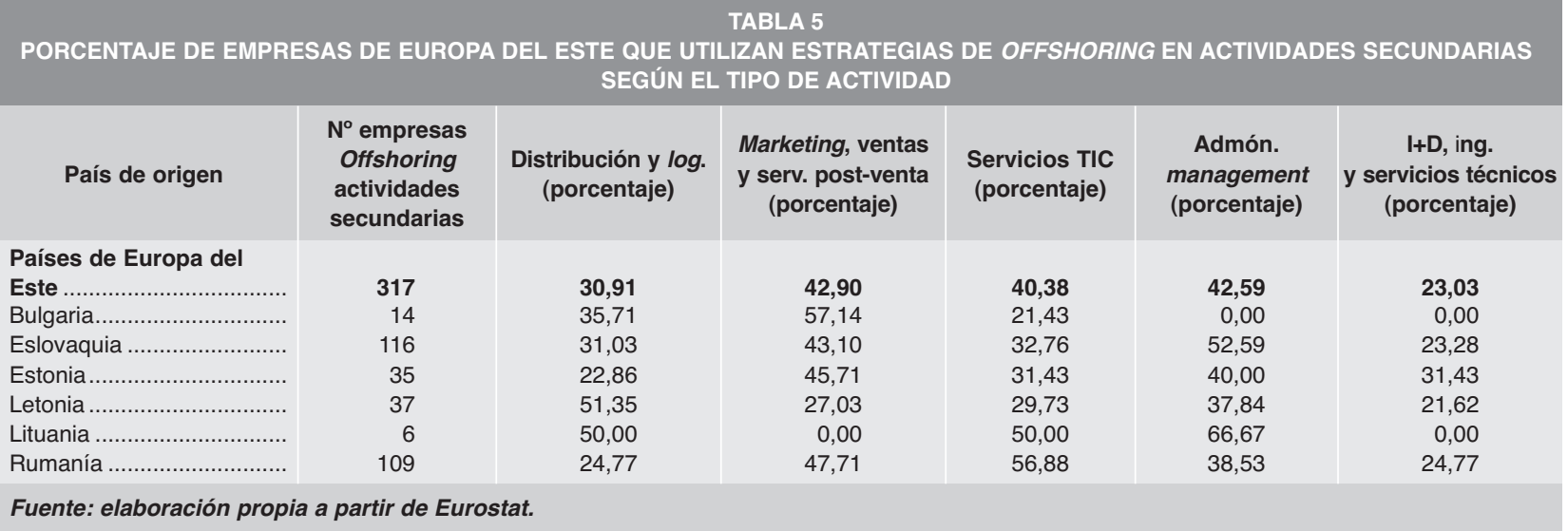

Si analizamos los datos a nivel de país, aunque en la mayoría de los países estudiados las empresas implementan estrategias de offshoring en actividades secundarias (entre el 74 y el 90 por 100), se puede observar cómo en el caso de Bulgaria el porcentaje de empresas que utilizan la estrategia para core activities coincide con el de aquellas que implementan offshoring en support activities (58,33 por 100).

Para identificar cuál es el tipo de actividad secundaria (support activities) que más se deslocaliza en los países del Este se debe analizar la Tabla 5. En ella puede observarse cómo las actividades comerciales (marketing, ventas y servicios post-venta) y las actividades administrativas (administración y management) son las deslocalizadas por un mayor número de empresas $(42,90$ y 42,59 por 100 , respectivamente). Otra de las actividades secundarias ampliamente deslocalizada por las empresas de estos países son los servicios TIC (40,38 por 100). Sin embargo, se observa cómo las empresas están menos dispuestas a deslocalizar actividades relacionadas con la I+D (23,03 por 100).

Si realizamos un análisis desagregado de los datos, observamos que existen importantes diferencias entre países. Así, por ejemplo, las empresas búlgaras deslocalizan en mayor medida actividades comerciales (57,14 por 100$)$; mientras que para la mayoría de las empresas eslovacas y lituanas las actividades administrativas son las preferidas para el uso de offshoring (52,59 y 66,67 por 100 , respectivamente). Destaca también el caso de Letonia en el que la actividad secundaria preferida por las empresas para su deslocalización es la distribución y logística (51,35 por 100); así como el caso de Rumanía en donde los servicios TIC son los deslocalizados en mayor medida (56,88 por 100$)$.

La modalidad de offshoring (IOF vs. EOF) utilizada por las empresas también puede ser distinta según el tipo de actividad deslocalizada (core vs. support). Las core activities suelen ser deslocalizadas a través de estrategias de IOF, ya que en la mayoría de los casos estas actividades están directamente relacionadas con la ventaja competitiva de la empresa y de este modo las empresas pueden mantener un mayor control sobre las mismas. Por otro lado, en la mayoría de las empresas la modalidad de offshoring utilizada para deslocalizar support activities es el EOF, motivado principalmente por el ahorro en costes que supone en aquellas actividades que no resultan básicas para mantener la ventaja competitiva de la empresa (Linares-Navarro, et al., 2014).

En el caso de las empresas analizadas (Gráfico 2), se observa cómo el IOF es la modalidad de offshoring más utilizada en los otros países europeos $(39,31$ vs. 18,36 por 100) para deslocalizar core activities, tal y como suele ser habitual. Sin embargo, resulta curioso que las empresas de los países de Europa del Este estudiados prefieran utilizar estrategias de EOF para deslocalizar este tipo de actividades $(21,78$ vs. 17,57 por 100). Dentro de estos países existen algunas particularidades a destacar como son el caso de $\square$ 

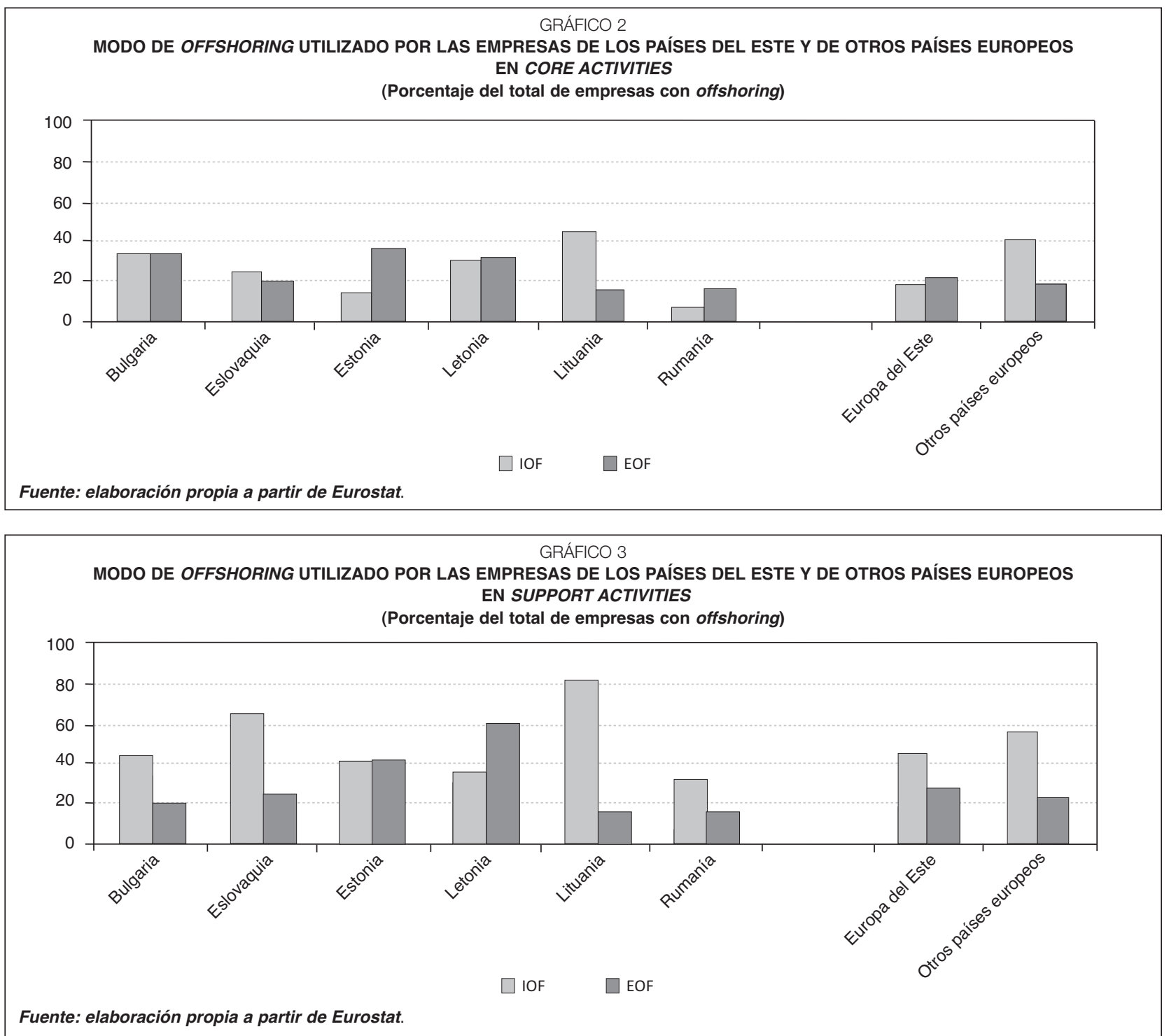

Eslovaquia o Lituania en los que la mayoría de las empresas utilizan IOF para deslocalizar actividades core. Destaca también el caso de Estonia en el que casi el 40 por 100 de las empresas utilizan EOF para deslocalizar este tipo de actividades.

Por otro lado, si analizamos la modalidad de offshoring utilizada por las empresas para deslocalizar support activities (Gráfico 3) observamos que, a diferencia de lo que suele ser habitual, en la mayoría de los países analizados la estrategia de offshoring utilizada para deslocalizar este tipo de actividades ha sido el IOF. Los casos de Lituania y Eslovaquia son los más significativos, ya que más del 80 por 100 y casi el 70 por 100 de las empresas respectivamente, utiliza esta modalidad para deslocalizar actividades secundarias. El único país en el que las empresas utilizan en mayor medida EOF para deslocalizar actividades secundarias es Letonia, en el que más del 60 por 100 de las empresas utiliza esta modalidad. Por último, destaca el caso de Estonia en el que el mismo porcentaje de empresas (42,22 por 100) utiliza las dos estrategias para deslocalizar este tipo de actividades.

\subsubsection{Offshoring vs. motivos}

Los motivos relacionados con costes son los que, generalmente, llevan a las empresas a realizar offshoring. Sin embargo, pueden existir motivos $\triangleright$ 


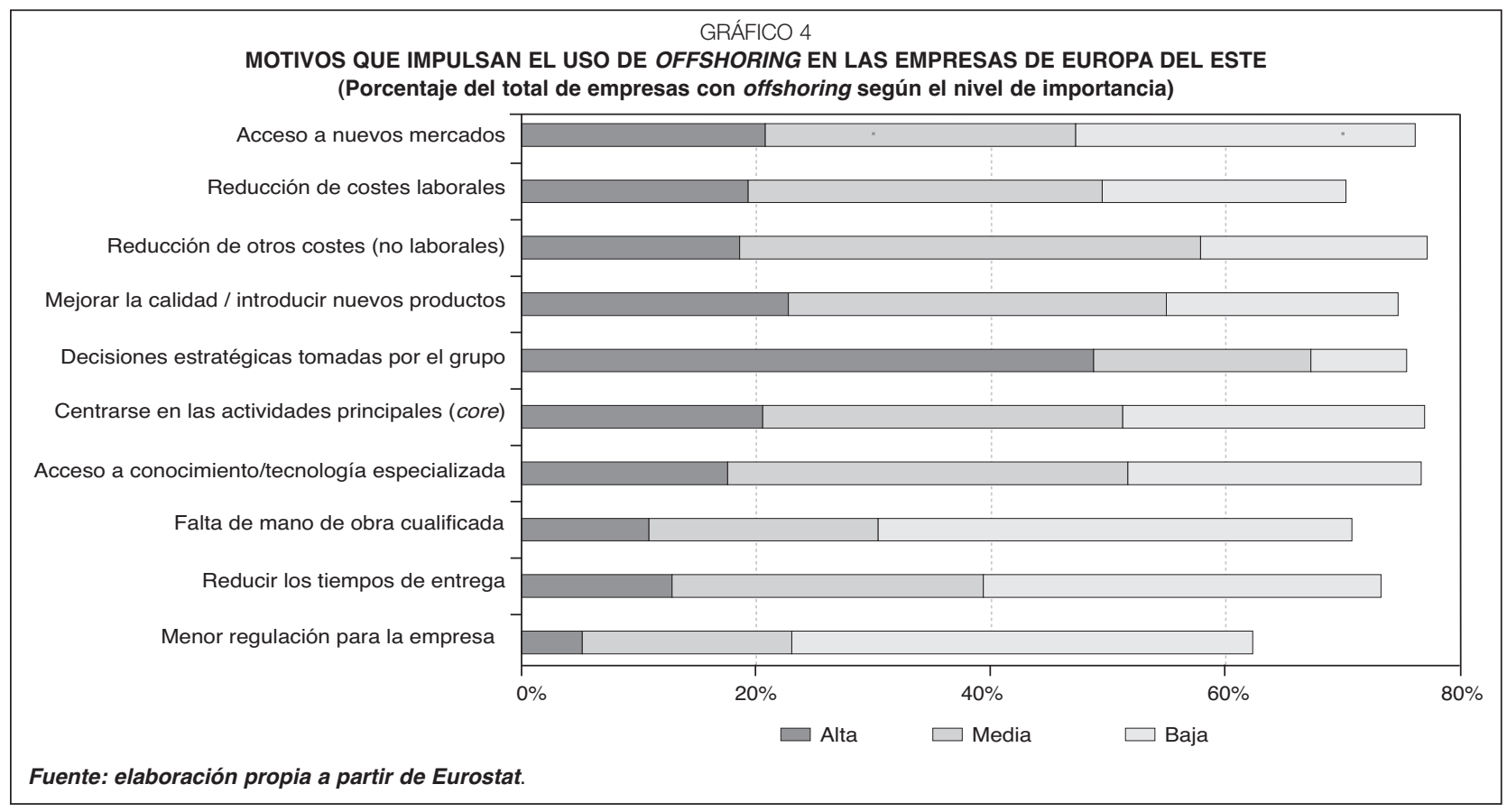

estratégicos que impulsen su uso (Linares, 2010). Algunas empresas las utilizan para acceder a mano de obra cualificada, mejorar la calidad de sus productos, o para centrarse en las competencias esenciales de su negocio. Otras, en cambio, responden a una decisión estratégica tomada por el grupo. Este último es el motivo principal por el que las empresas del este de Europa realizan offshoring (Gráfico 4). Sin embargo, para estas empresas el acceso a mano de obra cualificada, la reducción en los tiempos de entrega o una menor regulación -por ejemplo en materia medioambiental-, no son motivos relevantes.

Si observamos los datos según el país de origen (Gráfico 5), encontramos algunas diferencias. Mientras que para las empresas búlgaras el acceso a nuevos mercados es uno de los motivos principales; para las empresas eslovacas es más relevante la reducción de costes -laborales y no laborales-. Por otro lado, destaca que para las empresas lituanas el hecho de poder centrarse en las competencias esenciales sea uno de los motivos principales.

\subsubsection{Offshoring vs. destino}

Como ya se ha comentado, las empresas suelen deslocalizar sus actividades hacia países con niveles salariales más bajos, siendo éste uno de los elementos clave a la hora de elegir el país. Sin embargo existen otros criterios -de igual o mayor importancia-que las empresas también tienen en cuenta a la hora de elegir el país de destino del offshoring, como son la proximidad cultural y geográfica o la cualificación de la mano de obra. Los países asiáticos -principalmente China e Indiahan sido los destinos elegidos por numerosas empresas para deslocalizar sus actividades. Sin embargo, algunos países de la Unión Europea (UE) han sido considerados por las empresas igual o más importantes a la hora de realizar offshoring.

Si se analiza el Gráfico 6, en el que se recogen los principales destinos de las estrategias de offshoring utilizadas por empresas del este de Europa y de otros países europeos, se puede observar cómo la mayoría de las empresas europeas prefieren deslocalizar sus actividades dentro de la propia región (en países de la UE-15 o de la UE-12).

No obstante, si nos fijamos en los destinos preferidos por las empresas de cada uno de los dos grupos de países estudiados (países de Europa del Este vs. otros países europeos) observamos algunas diferencias. La mayoría de las empresas de los países del Este prefieren deslocalizar sus $\square$ 
GRÁFICO 5

MOTIVOS QUE IMPULSAN EL USO DE OFFSHORING EN LAS EMPRESAS DEL ESTE SEGÚN EL PAíS DE ORIGEN (Porcentaje del total de empresas con offshoring según el nivel de importancia)
BG:Bulgaria
SK: Eslovaquia
EE: Estonia
LV: Letonia
LT: Lituania
RO:Rumanía

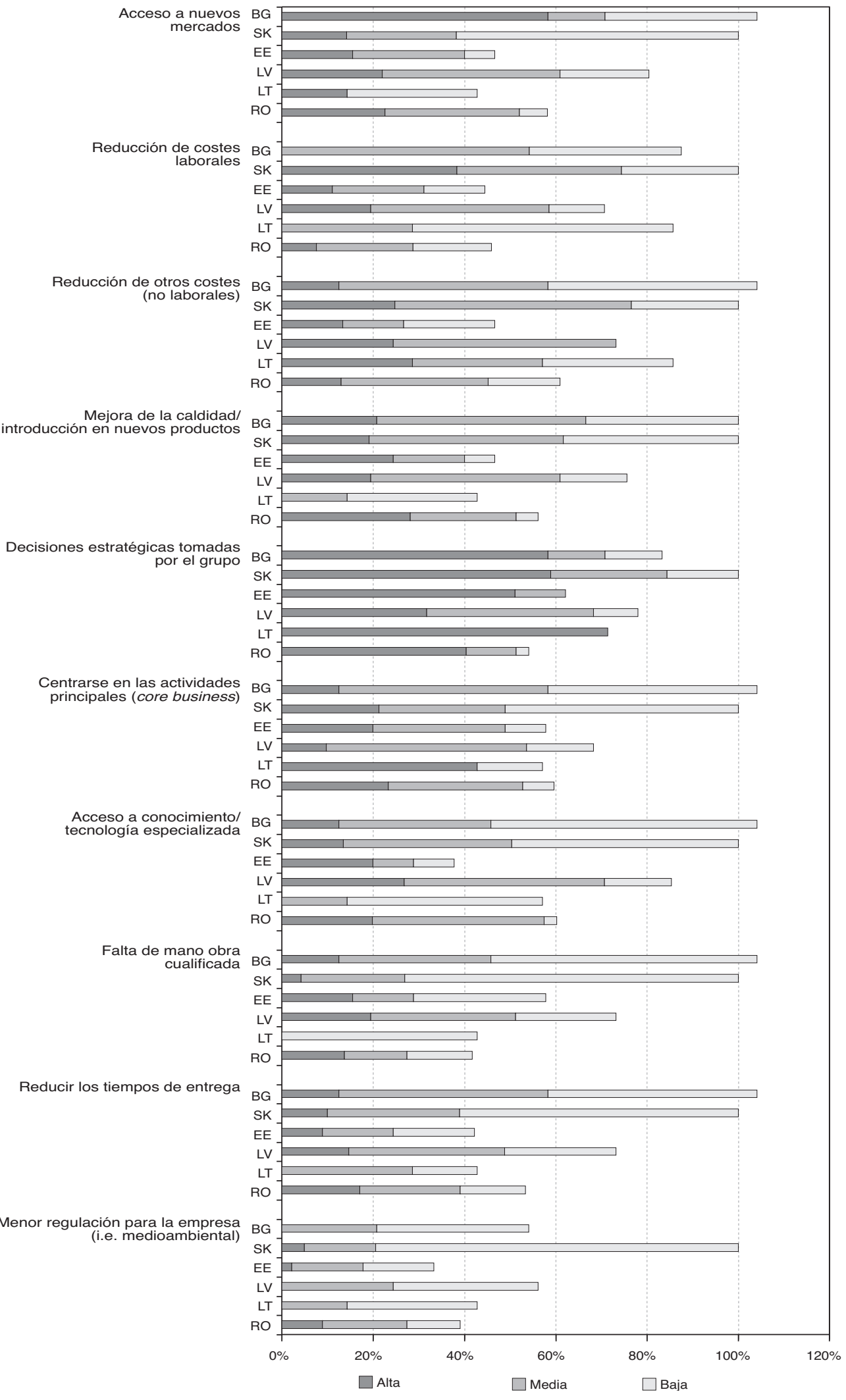

Fuente: elaboración propia a partir de Eurostat. 


\section{Esmeralda Linares Navarro}
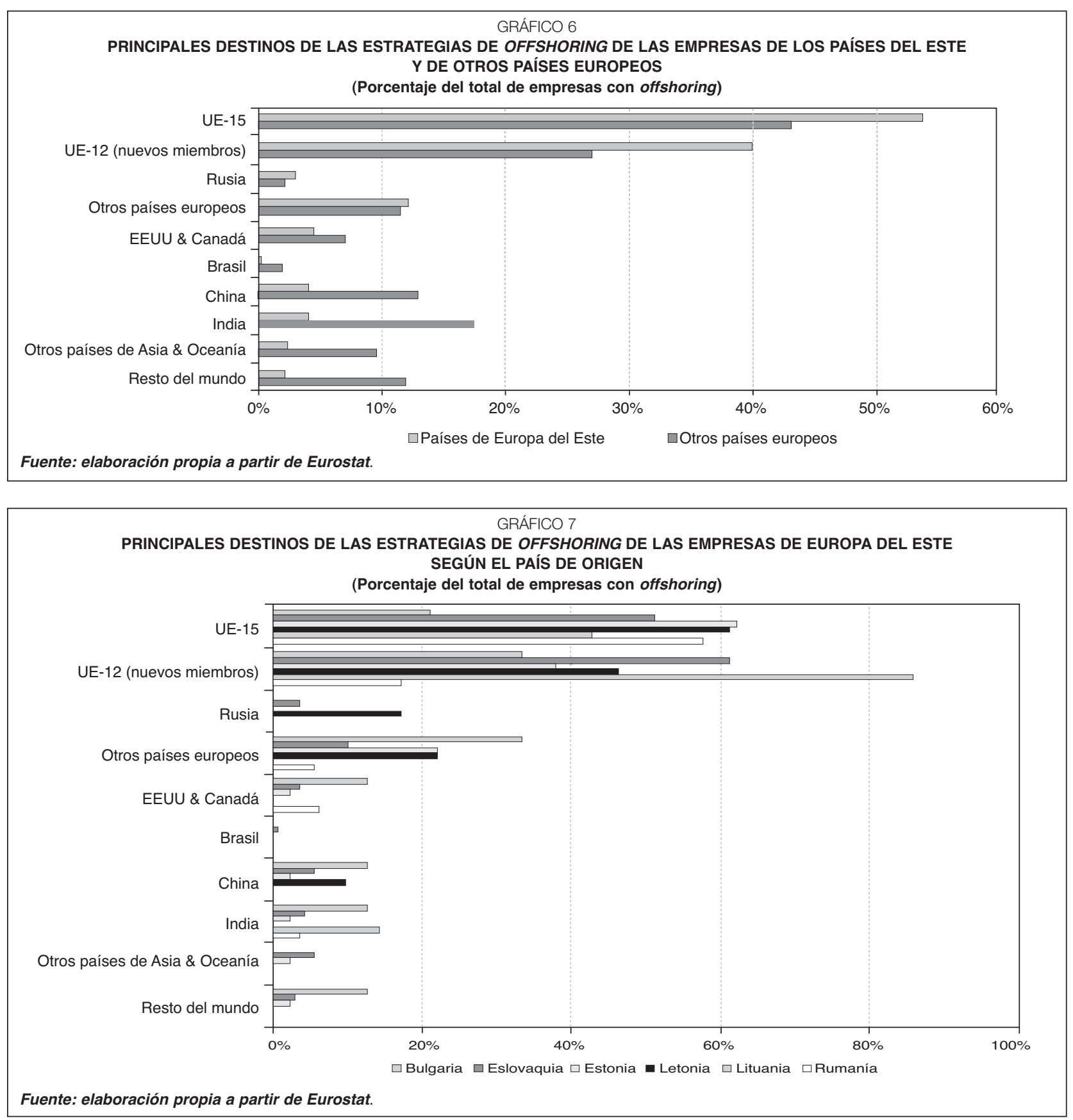

actividades dentro de Europa (un 53,71 por 100 en la UE-15; un 39,85 por 100 en la UE-12; y un 12,13 por 100 en otros países europeos); siendo el resto de destinos marginales (todos ellos inferiores al 5 por 100). Sin embargo, si analizamos los destinos elegidos por las empresas de "otros países europeos" observamos que aunque la mayoría de las empresas deslocalizan sus actividades dentro de la propia UE (un 43,02 por 100 en la UE-15 y un 26,79 por 100 en la UE-12); India y China son los siguientes países elegidos por estas empresas con un 17,20 y un 12,86 por 100 respectivamente.

Entre los países de Europa del Este estudiados también podemos encontrar importantes diferencias en la elección del país de destino (Gráfico 7). Prácticamente todos los países analizados prefieren la propia UE para deslocalizar sus actividades, sin embargo un elevado porcentaje de empresas búlgaras utilizan otros países europeos $\triangleright$ 


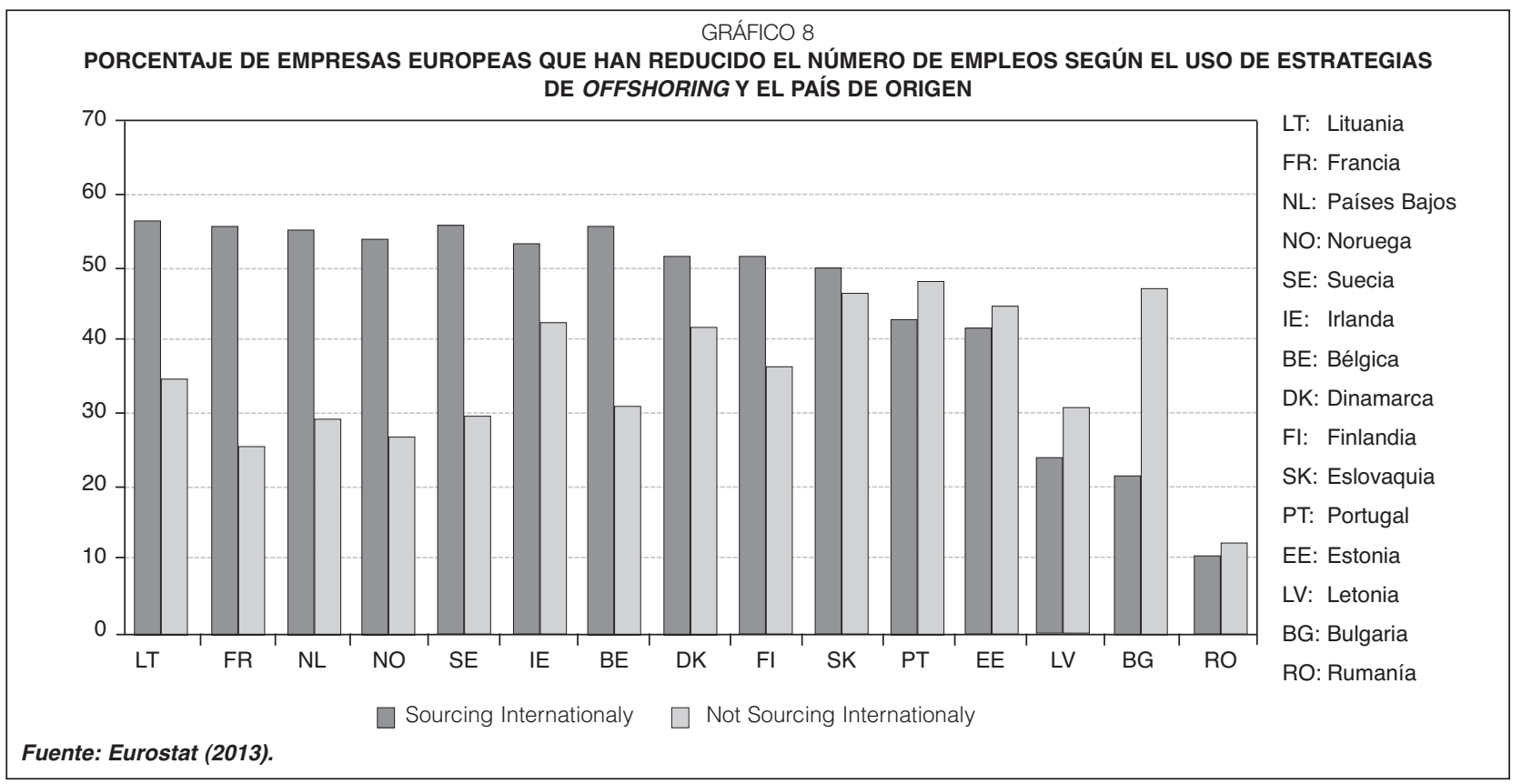

para realizar offshoring $(33,33$ por 100$)$, así como otros países no europeos, como son EEUU y Canadá, China o India (un 12,50 por 100 en todos ellos). Por otro lado, destaca también el caso de Letonia donde un 17,07 por 100 de las empresas eligen Rusia y casi un 10 por 100 utiliza China como destino del offshoring. Otro caso destacable es el de Lituania, donde el 14,29 por 100 de las empresas utiliza la India para deslocalizar sus actividades y casi el 86 por 100 de sus empresas prefieren los países de la UE-12 para llevar a cabo el offshoring.

\subsubsection{Offshoring vs. empleo}

Aunque las estrategias de offshoring poseen numerosas ventajas para aquellas empresas que deciden llevarlas a cabo, no cabe duda que el uso de estas estrategias por parte de las empresas influirá en los niveles de empleo del país de origen de estas empresas. La reducción en los niveles de empleo que se suele dar en estos países se ha considerado como uno de los principales problemas de la estrategia. Sin embargo, la influencia del offshoring sobre el empleo del país de origen puede no resultar tan negativa como parece, llegando en algunos casos a favorecer los niveles de empleo altamente cualificado de algunos países (Linares, 2010).

Los efectos del offshoring sobre los niveles de empleo no afectan por igual a todos los países. En el Gráfico 8 se observa cómo un elevado porcentaje de empresas europeas han reducido el número de empleos a causa del mismo. Sin embargo, en la mayoría de los países de Europa del Este la disminución de empleos se ha originado en mayor medida por empresas que no utilizan offshoring, por lo que el efecto negativo del uso de la estrategia sobre el empleo de estos países es menor.

Si se analiza el número de empleos perdidos en los países del este de Europa como consecuencia del offshoring (Gráfico 9, en la página siguiente) se observa que Eslovaquia es el país que más empleos ha destruido (2.501), mientras que Lituania y Rumania son los dos países que menor número de empleos han perdido (63 y 193 , respectivamente). El resto de países presentan valores próximos a los 400 empleos. Por otro lado, si analizamos los empleos perdidos según el tipo de actividad deslocalizada (core vs. support activities), destaca que el mayor número de empleos perdidos en casi todos los países se ha dado en actividades principales (core activities). 


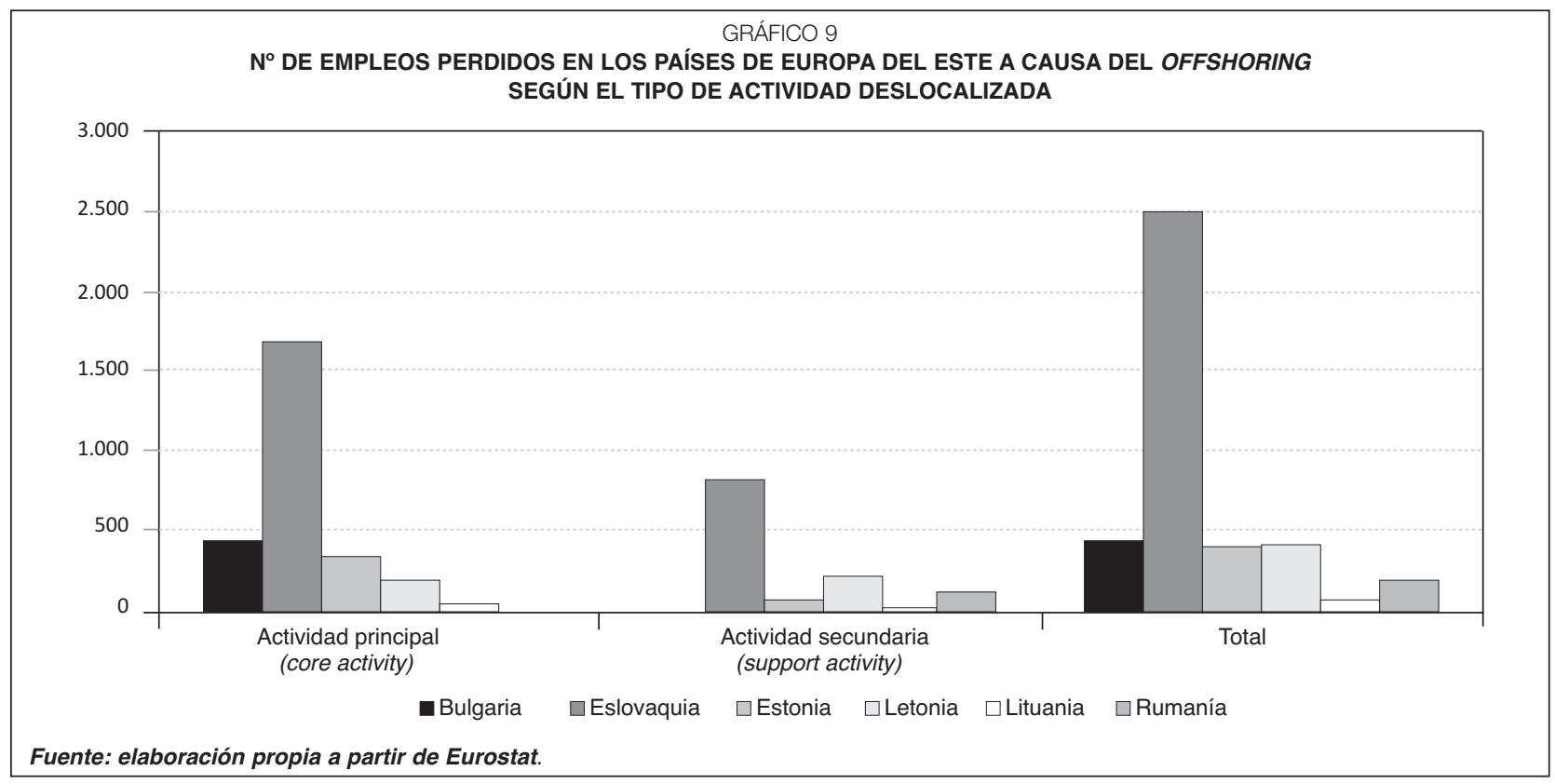

\section{Conclusiones}

Los beneficios derivados del offshoring -una de las estrategias más utilizadas por las empresas en los últimos años-, así como las características del entorno actual, han llevado a numerosas empresas a deslocalizar sus actividades en distintas partes del mundo. Después de India y China, los países de Europa del Este han sido los preferidos por muchas empresas para la deslocalización de sus actividades. No obstante, en estos países también existen empresas que utilizan el offshoring como vía para mantener su ventaja competitiva. El estudio de esta región europea como destino elegido por las empresas para llevar a cabo estas estrategias ha sido objeto de numerosas investigaciones (Marin, 2006; Sass y Fifekova, 2011; Stare y Rubalcaba, 2009); sin embargo, el análisis de Europa del Este como «origen del offshoring» prácticamente no ha sido estudiado.

A través de los datos de la International Sourcing Survey (Eurostat, 2012), en la que se recoge información sobre las estrategias de offshoring llevadas a cabo por empresas europeas, en este trabajo se analizan las principales características del uso de la estrategia en algunos países del este de Europa identificando diferencias entre las características básicas de la estrategia y las propias de estos países.

Entre las características básicas de las estrategias de offshoring se encuentran las que hacen referencia al sector de actividad y a la modalidad implementada. Las empresas del sector manufacturero suelen ser las protagonistas del uso de estas estrategias (Eurostat, 2013); y el EOF el modo de offshoring más utilizado. El uso de estas estrategias también suele ser distinto según el tipo de actividad. Las support activities suelen ser las actividades más propensas a la deslocalización y en la mayoría de los casos a través de EOF; sin embargo, es cada vez más frecuente que se deslocalicen core activities, fundamentalmente a través de IOF (Linares-Navarro et al., 2014). Por otro lado, entre las razones que impulsan a las empresas a realizar offshoring, el ahorro en costes -esencialmente laborales- suele ser el motivo principal por el que la mayoría de las empresas utiliza la estrategia. Éste es, además, uno de los criterios que se tiene en cuenta para elegir el país de destino del offshoring, aunque en ocasiones la proximidad cultural y/o geográfica resulte igual o más importante. Por último, uno de los principales problemas asociados es la reducción en los niveles de empleo del país de origen. No $\square$ 
obstante, la influencia del offshoring sobre el empleo puede no resultar tan negativa como parece (Linares, 2010).

En general, en los países del este de Europa la estrategia de offshoring está siendo ampliamente utilizada tanto por empresas manufactureras como de servicios, lo que contrasta con lo ocurrido en la mayoría de los países europeos donde son las manufacturas las que mayor uso hacen. En cuanto a la modalidad de offshoring, en esta región predomina el IOF, algo totalmente distinto a lo que ocurre en la mayoría de los casos, donde el EOF es la estrategia mayoritaria. Por otro lado, en Europa del Este las empresas deslocalizan en mayor medida support activities; sin embargo lo hacen utilizando IOF, algo poco habitual. Destaca también que el modo preferido por estas empresas para deslocalizar core activities sea el EOF, ya que en estos casos la mayoría de las empresas suelen utilizar IOF con el fin de preservarlas. Otro aspecto a destacar en las empresas del este de Europa es la razón principal por la que realizan offshoring. Suele ser habitual que los costes sean uno de los motivos principales por el que las empresas deciden utilizarlo. Sin embargo, para la mayoría de las empresas de esta región el uso de estrategias de offshoring se debe a una decisión estratégica tomada por el grupo. En cuanto al destino elegido, parece que la proximidad cultural y geográfica tiene mucha importancia para la mayoría de las empresas de esta región, ya que éstas prefieren deslocalizar sus actividades dentro de la propia Unión Europea, siendo marginal la elección de otros países. Por último, en Europa del Este el efecto negativo del uso del offshoring sobre el empleo es menor al del resto de países europeos, ya que la mayor disminución de empleos en estos países la han originado empresas que no utilizan la estrategia.

Los resultados de esta investigación ponen en evidencia que el uso de estas estrategias en Europa del Este presenta características distintas, permitiendo avanzar en su ámbito de estudio. Sin embargo, el reducido tamaño de la muestra o el limitado número de países examinados son dos de las principales limitaciones de este trabajo que deberían ser superadas en futuras investigaciones.

\section{Bibliografía}

[1] AMESSE, F.; DRAGOSTE, L.; NOLLET, J. y PONCE, S. (2001). Issues on partnering: evidences from subcontracting in aeronautics. Technovation, vol. 21, n 9,pp. 559-569.

[2] AT KEARNEY (2014). A wealth of choices: from anywhere on earth to no location at all. The 2014 A.T. Kearney Global Services Location Index.

[3] DESS, G.; RASHEED, A.; MCLAUGHLIN, K. y PRIEM, R. (1995). The new corporate architecture. Academy of Management Executive, $\mathrm{n}^{\circ}$ 9, pp. 7-20.

[4] EUROSTAT (2009). International Sourcing in Europe - Statistics in focus 4/2009. Eurostat.

[5] EUROSTAT (2012). International sourcing statistics. Structural Business Statistics. Disponible en:

http://ec.europa.eu/eurostat/data/database (Recuperado el 2 de diciembre de2014)

[6] EUROSTAT (2013). International Sourcing of business functions. Statistics Explained, June 2013. Eurostat.

[7] JENSEN, P. D. Ø.; y PEDERSEN, T. (2011). «The economic geography of offshoring: the fit between activities and local context». Journal of Management Studies, vol. $48, n^{\circ} 2$, pp. 352-372.

[8] KOTABE, M.; MOL, M. J. y MURRAY, J. Y. (2008). "Outsourcing, performance, and the role of e-commerce: A dynamic perspective». Industrial Marketing Management, vol. 37, $\mathrm{n}^{\circ}$ 1, pp. 37-45.

[9] KOTABE, M. y MURRAY, J. Y. (1990). «Linking product and process innovations and modes of international sourcing in global competition: A case of foreign multinational firms». Journal of International Business Studies, $\mathrm{n}^{\circ} 21$, pp. 383-408.

[10] KOTABE, M. y MURRAY, J.Y. (2004). «Global Sourcing strategy and sustainable competitive advantage». Industrial Marketing Management, $\mathrm{n}^{\circ}$ 33, pp. 7-14.

[11] LEVY, D. (2015). «Offshoring in the new global political economy». Journal of Management Studies, vol. 42, n 3, pp. 685-693.

[12] LINARES, E. (2010). La Deslocalización Industrial en Europa. El fenómeno del Offshoring. Analistas Económicos de Andalucía. 
[13] LINARES-NAVARRO, E.; PEDERSEN, T. y PLA-BARBER, J. (2014). «Fine slicing of the value chain and offshoring of essential activities: empirical evidence from European multinationals». Journal of Business Economics and Management, vol. $15, n^{\circ} 1$, pp. 111-134.

[14] LISLE, C. (2003). "Outsource a core competency? Why private equity groups are outsourcing business strategy due diligence». Journal of Private Equity, vol. 7, $\mathrm{n}^{\circ}$ 1, pp. $72-76$.

[15] MARIN, D. (2006). «A new international division of labor in Europe: Outsourcing and offshoring to Eastern Europe». Journal of the European Economic Association, vol. 4, no 2-3, pp. 612-622.

[16] OCDE (2007). Offshoring and Employment: Trends and Impacts. OECD 2007.

[17] PAGNONCELLI, D. (1993). «Managed outsourcing: a strategy for a competitive company in the 1990s». Management Decision, $\mathrm{n}^{\circ}$ 31, pp. 15-22.

[18] PYNDT, J. y PEDERSEN, T. (2006). Managing global offshoring strategies: A case approach. Copenhagen Business School Press DK.

[19] QUINN, J.B. (1992). Intelligent enterprise: A knowledge and service based paradigm for industry. New York: Free Press.
[20] ROZA, M.; VAN DEN BOSCH, F. A. y VOLBERDA, H. W. (2011). "Offshoring strategy: Motives, functions, locations, and governance modes of small, medium-sized and large firms». International Business Review, vol. 20, $\mathrm{n}^{\circ} 3$, pp. 314-323.

[21] SASS, M. y FIFEKOVA, M. (2011). "Offshoring and outsourcing business services to Central and Eastern Europe: Some empirical and conceptual considerations». European Planning Studies, vol. 19, n 9, pp. 1593-1609.

[22] SCHNIEDERJANS, M.J. y ZUCKWEILER, K.M. (2004). «A quantitative approach to the outsourcing-insourcing decision in an international context». Management Decision, vol. 42, $\mathrm{n}^{\circ} 8$, pp. 974-986.

[23] STARE, M. y RUBALCABA, L. (2009). «International Outsourcing of Services: What Role for Central and East European Countries?» Emerging Markets Finance and Trade, vol. 45, $\mathrm{n}^{\circ} 5$, pp. 31-46.

[24] WTO (2005). Standards, 'offshoring' and air transport focus of 2005 WTR. World Trade Report.

[25] ZSIDISH, G.A. (2003). "Managerial perspective on supply risk». Journal of Supply Chain Management, vol. 39, $\mathrm{n}^{\circ}$ 1, pp. 14-26. 